\title{
Factores que favorecen el desarrollo de una actitud positiva hacia las actividades académicas
}

\author{
Factors which favor the development of a positive \\ attitude towards academic activities
}

\author{
María Luisa Naranjo Pereira \\ Docente de la Escuela de Orientación y Educación Especial \\ Universidad de Costa Rica \\ San José, Costa Rica \\ malunape@gmail.com
}

Recibido 24-01-2010 • Aceptado 12-04-2010 • Corregido 05-05-2010

Resumen: Este artículo presenta una investigación de tipo descriptivo, cuyo objetivo es analizar los factores personales que favorecen el desarrollo de una actitud positiva hacia las actividades académicas, de acuerdo con la opinión de dos grupos de estudiantes de décimo año. Ella está constituida por un constructo principal: la actitud positiva hacia las actividades académicas, y un subconstructo: los factores personales que favorecen esas actitudes. Los aspectos que integran este subconstructo son la autoestima positiva, la motivación, la comunicación asertiva y el manejo del estrés. La introducción y el referente teórico evidencian la relación que existe entre estos factores, las actitudes hacia el aprendizaje y los logros académicos de la población estudiantil. De acuerdo con los resultados obtenidos, se concluye que la población estudiantil concede importancia tanto a factores internos como externos relacionados con la motivación, y los percibe como significativos para el desarrollo de actitudes positivas hacia las actividades académicas. De igual forma sucede con el hecho de poseer una autoestima positiva, saber comunicarse asertivamente y poder manejar el estrés de forma adecuada. Se recomienda, por lo tanto, que las instituciones educativas presten especial atención al desarrollo socio-emocional del estudiantado, mediante la realización de programas que favorezcan el logro de su motivación académica, un mejoramiento de su autoestima, la habilidad

\section{Introducción}

En el sistema educativo, un aspecto relevante es que cada estudiante, como individualidad, no se pierda entre la multitud de una educación en masa y que se le ayude a desarrollar sus facultades potenciales. Es probable que su desempeño esté influenciado por una serie de factores personales, que pueden afectar positiva o negativamente sus actitudes hacia las actividades educativas y por ende sus logros académicos.

Para que una institución educativa sea efectiva, debe hacer énfasis tanto en el desarrollo socio-emocional de cada estudiante, como en su desarrollo cognitivo. Estudiar de qué manera los factores internos de la persona favorecen el desarrollo de actitudes positivas hacia las actividades académicas es el tema de esta investigación, por lo que se realiza una revisión de aspectos como la motivación, la autoestima positiva, la comunicación asertiva y el manejo del estrés. 
para comunicarse asertivamente y el afrontamiento apropiado del estrés.

Palabras clave: Actitudes, motivación, autoestima, asertividad, educación.

\begin{abstract}
This paper is a descriptive investigation whose objective is to analyze personal factors which favor the development of a positive attitude towards academic activities, according to the opinion of two groups of tenth grade students. The investigation consists of a primary construct: a positive attitude towards academic activities, and a sub-construct: personal factors which promote such attitudes. The aspects which make up this sub-construct are positive self-esteem, motivation, assertive communication and stress management. The introduction and theoretical references provide evidence for the relationship between these factors, attitudes towards learning and student academic achievements. In agreement with the obtained results, it is concluded that the student population attaches importance to both internal and external factors related to motivation, and perceives them as significant for the development of a positive attitude towards academic activities. The same holds true for having positive self-esteem, knowing how to communicate assertively and being able to manage stress appropriately. Therefore, it is recommended that educational institutions give special attention to a student's socio-emotional development by implementing programs which promote motivation for academic achievement, improved self-esteem, the ability to communicate assertively and appropriate coping methods for stress.
\end{abstract}

Key words: Attitudes, motivation, self esteem, assertiveness, education.
Sobre el tema de la motivación, Krauskopf (1989) considera que la institución educativa debe mostrar actitudes de apoyo, comprensión y ayuda, por cuanto el tipo de relaciones interpersonales entre el personal docente y el estudiantado ejerce influencia en el rendimiento académico de este, ya que si se siente apoyado y que se comprenden sus dificultades, puede mantener o aumentar su motivación hacia el aprendizaje. Por el contrario, si la posición del profesorado es de hostilidad o inflexibilidad, mostrará probablemente una actitud defensiva, lo cual puede afectar negativamente su motivación hacia las actividades académicas.

Respecto al estrés, Cascante y Salazar (1993) señalan que el estrés entorpece la labor académica del estudiantado principalmente durante las pruebas, por lo que se considera importante el apoyo que se le brinde, pues le ayuda a enfrentar de manera más adecuada el efecto estresante que pueden tener las distintas actividades académicas.

Santrock (2002) explica que la ansiedad es un sentimiento desagradable y vago, de temor y aprehensión. Se ha encontrado que mucha de la población estudiantil manifiesta niveles moderados de ansiedad; no obstante, cierta parte de esa población presenta niveles muy elevados de ansiedad, con preocupaciones constantes, lo cual puede perjudicar de manera significativa su rendimiento y su habilidad de logro. Es importante por lo tanto tomar en cuenta el efecto de los niveles de tensión y de ansiedad en el desempeño de las actividades educativas.

Algunos resultados importantes que se obtuvieron en la investigación realizada por Cascante y Salazar (1993) son que el nivel de estrés aumenta conforme la población estudiantil avanza en el nivel educativo, por cuanto se tiende a una mayor exigencia académica en los niveles superiores.

En cuanto a la autoestima, se ha encontrado que esta tiene una influencia relevante sobre las competencias sociales y la resistencia al estrés. Además, según Harter (1999), una autoestima baja puede 
conducir a otros problemas más serios, tales como la dificultad para establecer relaciones interpersonales adecuadas, ansiedad social, depresión e incluso delincuencia, lo cual le impide al estudiante lograr el apoyo necesario de otras personas.

Según lo menciona Santrock (2002), las habilidades sociales le permiten a la persona abrirse hacia las demás y ser más asertiva en las relaciones interpersonales, lo cual, a la vez, le genera mayores recursos para afrontar situaciones estresantes.

De acuerdo con lo analizado, parece existir una relación estrecha entre los aspectos considerados, a saber, motivación, autoestima, habilidades de comunicación y afrontamiento del estrés, que repercute en las actitudes de la población estudiantil respecto de las actividades académicas, lo que a su vez se refleja en los niveles de desempeño educativo.

A partir de lo planteado, surge la inquietud de investigar acerca de los factores personales que favorecen el desarrollo de actitudes positivas hacia las actividades académicas en estudiantes de educación secundaria. La investigación se realizó en el año 2007 con dos grupos de estudiantes de décimo año. Los resultados pueden ser un aporte para que desde las instituciones educativas se trabaje con estos aspectos en beneficio de la población estudiantil y en general de la calidad de la educación.

\section{Objetivo general}

Analizar los factores personales que favorecen el desarrollo de una actitud positiva hacia las actividades académicas de acuerdo con la opinión de dos grupos de estudiantes de décimo año.

\section{Objetivos específicos}

- Identificar los aspectos relacionados con la autoestima que favorecen el desarrollo de una actitud positiva hacia las actividades académicas.

- Identificar los aspectos relacionados con la motivación que favorecen el desarrollo de una actitud positiva hacia las actividades académicas.

- Identificar los aspectos relacionados con la comunicación asertiva que favorecen el desarrollo de una actitud positiva hacia las actividades académicas.

- Identificar los aspectos relacionados con el manejo del estrés que favorecen el desarrollo de una actitud positiva hacia las actividades académicas.

\section{Referente teórico}

\section{Actitudes hacia las actividades académicas}

Cárdenas (2008) considera que la actitud es una predisposición positiva o negativa que influye en el comportamiento de la persona. Está conformada por tres componentes: el cognitivo, esto es, las creencias que subyacen en esa actitud; el afectivo, relacionado con sentimientos de aceptación o de rechazo y el intencional que se manifiesta en la tendencia hacia determinado comportamiento.

De acuerdo con el sitio web definición.de (2008), la actitud es una predisposición aprendida para responder de manera consistente a un objeto social. Se distinguen tres componentes de las actitudes: el cognoscitivo, formado por las percepciones y creencias que la persona tiene hacia un objeto social, así como por la información que se tiene sobre este; el afectivo, es decir, el sentimiento que surge a favor o en contra del objeto social, y el conductual, que se refiere a la tendencia a reaccionar hacia los objetos de cierta manera.

En opinión de Valera (2001), las actitudes son principalmente objetos a construir mediante la educación. Su construcción debe estimularse a partir de tres 
dimensiones: la cognitiva, entendida como conocimiento, creencias e ideas; la afectiva, entendida como los sentimientos que valoran un objeto o conducta de forma negativa o positiva y, la conductual, es decir, la intención que evidencia la persona para la acción.

Zeledón y Chavarría (2001) mencionan algunas características básicas de las actitudes, de las cuales se nombran algunas a continuación. Por un lado, son adquiridas: se trata de respuestas electivas ante los valores construidos mediante un proceso cognitivo, las cuales son posibles si la razón acepta el valor. Asimismo, son flexibles: susceptibles de cambio. También son cualidades perceptibles: con capacidad de crecimiento, pueden arraigarse, deteriorarse o perderse. Igualmente, son motivacionales: entrañan procesos afectivos y volitivos; esto es, las actitudes alcanzan valores mediante los deseos, la sensibilidad y la voluntad. Por otro lado, son complejas o integrales: se presentan en la persona de manera correlacionada en sus componentes, a saber, cognitivo, afectivo y conativo. Además de esto, son transferibles: se pueden actualizar de modos diversos y hacia distintos objetivos.

Existen dos formas de adquirir actitudes, una es de modo directo, cuando la persona lo hace mediante su propia experiencia, lo cual le produce satisfacción y significado. La otra es de modo indirecto, cuando la persona adquiere la actitud por medio de la identificación con otras a las que respeta y admira, quienes se convierten en modelos auténticos.

En cuanto a las actitudes favorables hacia las actividades académicas, estas deben estar inmersas en el proceso de desarrollo del aspecto formativo de la población estudiantil.

Las actitudes positivas del estudiantado hacia las actividades académicas y el apoyo de otros significativos, por ejemplo la familia, pares y personal docente, tienen una importante influencia sobre los autoconceptos académicos de las personas estudiantes. Cuando estas sienten que otras personas confían en sus capacidades académicas, experimentan confianza en sí mismas.

Rice (2000) menciona que estudiantes con actitudes negativas, que incluso llegan a abandonar sus estudios, pueden mostrar síntomas de un mal funcionamiento del yo; por ejemplo: baja autoestima, sentimientos de inferioridad, excesivo miedo y ansiedad o inestabilidad emocional.

Stevens y Phil, citados por este autor, indican que se han identificado cinco rasgos comunes entre quienes tienen un bajo rendimiento académico: negativismo, sentimientos de inferioridad, alta ansiedad, aburrimiento y sobre-protección. Cuando se enfrentan a obstáculos para conseguir el éxito, muestran actitudes negativas, tienen menos tendencia a creer que van a poder lograr su objetivo y a buscar una ayuda adecuada. Presentan mayor tendencia a sentirse desvalidos, a centrarse en su falta de capacidad y a poner en marcha estrategias que son poco eficaces.

Sobre este mismo tema, Jackson y Frick (1998) señalan que cuando el estudiantado llega a concluir que las demandas del centro educativo son más de lo que puede rendir al realizar su evaluación sobre ellas, este afronta una situación de humillación, a veces suficiente para justificar actitudes negativas y un rechazo a la institución.

De acuerdo con lo analizado, las actitudes son factores que tienen gran influencia en el comportamiento de las personas. Siendo esto así, las actitudes de la población estudiantil hacia las actividades académicas pueden tener un impacto positivo o negativo que repercute en la calidad de su proceso de aprendizaje y en su bienestar personal general.

\section{Motivación}

Ajello (2003) señala que la motivación debe ser entendida como la trama que sostiene el desarrollo de aquellas actividades que son significativas para la persona 
y en las que esta toma parte. En el plano educativo, la motivación debe ser considerada como la disposición positiva para aprender y continuar haciéndolo de una forma autónoma.

De acuerdo con Santrock (2002), existen tres perspectivas fundamentales respecto de la motivación: la conductista, la humanista y la cognitiva. La conductista subraya el papel de las recompensas en la motivación, la humanista se enfoca en las capacidades del ser humano para desarrollarse y la cognitiva enfatiza en el poder del pensamiento.

\section{Perspectiva conductual}

Esta perspectiva señala que las recompensas externas y los castigos son centrales en la determinación de la motivación de las personas (Santrock, 2002). Las recompensas son eventos positivos o negativos que pueden motivar el comportamiento.

Skinner (1977), citado en Trechera (2005), explica que las teorías que se basan en el empleo de incentivos parten del supuesto de que:

... Las personas suelen realizar comportamientos con el objetivo de obtener algún beneficio y evitan o dejan de hacer aquellas conductas que conllevan un daño. Para este enfoque toda modificación de conducta se realiza básicamente a través de refuerzos, recompensas o mediante la evitación u omisión de aquello que sea desagradable. (p. 3)

\section{Perspectiva humanista}

La perspectiva humanista se enfatiza en la capacidad de la persona para lograr su crecimiento, sus características positivas y la libertad para elegir su destino. De acuerdo con García (2008), una de las teorías más conocidas sobre la motivación es la de la jerarquía de las necesidades, propuesta por Abraham H. Maslow, quien concibió las necesidades humanas ordenadas según una jerarquía, donde unas son prioritarias y sólo cuando están cubiertas se puede ascender a necesidades de orden superior. Una vez satisfechas una serie de necesidades, estas dejan de fungir como motivadores. De acuerdo con esta teoría las necesidades se satisfacen en el siguiente orden: necesidades fisiológicas, de seguridad, de amor y pertenencia (sociales), de estima y de autorrealización.

Existen otras teorías clásicas de la motivación que han evolucionado a partir de la teoría de Maslow. Por ejemplo, la teoría Existencia, Relación y Crecimiento (E.R.G.) desarrollada por Alderfer, 1972 (citado por Trechera, 2005). Este autor agrupa las necesidades en tres tipos:

Existencia: son aquellas necesidades básicas y materiales que se satisfacen mediante factores externos y corresponden a las necesidades fisiológicas y de seguridad.

Relación: requieren para su satisfacción de las relaciones interpersonales y de la pertenencia a un grupo. Se refieren a las necesidades sociales y de aceptación.

Crecimiento: son las necesidades de desarrollo personal. Se satisfacen cuando la persona logra objetivos importantes para su proyecto vital, e incluyen el reconocimiento, la autoestima y la autorealización.

Hampton, Summer y Webber (1989) mencionan que la teoría de las necesidades de Mc Clelland señala que existen tres motivaciones importantes: la necesidad de logro, la de afiliación y la de poder. Estas son importantes porque predisponen a las personas a comportarse en formas que afectan de manera crítica el desempeño en muchos trabajos y tareas.

Autores como Valdés (2005), Trechera (2005) y García (2008) mencionan que a las personas con una alta necesidad de logro les gustan las situaciones en las que pueden tomar la responsabilidad de encontrar la solución a los problemas; tienden a fijarse metas moderadas y a tomar riesgos calculados; desean retroalimentación acerca de qué tan adecuadamente se están desempeñando y se distinguen por intentar 
hacer bien las cosas, tener éxito, incluso por encima de las recompensas

Sobre la necesidad de poder, las personas que tienen una alta necesidad de este siempre necesitan ganar argumentos, persuadir a otras, hacer que sus criterios prevalezcan y se sienten incómodas si no poseen cierta cuota de autoridad. En cuanto a la necesidad de afiliación, las personas con una alta necesidad de esta tienden a interesarse y a pensar con frecuencia acerca de la calidad de sus relaciones personales. La meta común es la interacción social y lograr la aceptación de otras personas.

La inclusión, aceptación y aprobación del grupo de iguales, específicamente en estudiantes, tiene una marcada influencia sobre la motivación de logro. Esa pertenencia se define como el sentimiento que tiene la persona de ser valorada, incluida y motivada por otras (Rice, 2000).

Bandura (1994) se refiere al concepto de autosuficiencia como la creencia que tiene la persona de que puede dominar una situación y lograr resultados positivos. Considera que es un factor determinante en el éxito académico del estudiantado. Es probable que las personas con baja autosuficiencia en el aprendizaje eviten ciertas tareas, sobre todo las que son desafiantes, mientras que aquellas con alta autosuficiencia trabajen con entusiasmo en actividades de aprendizaje. Sentirse autosuficiente en una variedad de situaciones ofrece como recompensa una mejoría en la autoestima y la motivación.

\section{Perspectiva cognitiva}

Santrock (2002) indica que, de acuerdo con la perspectiva cognitiva, los pensamientos, en el caso concreto de la persona estudiante, guían su motivación. La teoría cognitiva se centra en ideas tales como la motivación interna de logro de las personas, sus atribuciones acerca del éxito o del fracaso, sus creencias sobre lo que pueden controlar de forma efectiva en su ambiente, la importancia del establecimiento de metas, el planeamiento y el monitoreo del progreso hacia una meta.

Valdés (2005); García (2008) y Trechera (2005) señalan que la Teoría de las Expectativas fue elaborada por Víctor Vroom, quien considera que las personas se motivan a realizar cosas y esforzarse por lograr un alto desempeño para alcanzar una meta si creen en su valor, si están seguras de que lo que harán contribuirá a lograrla y si saben que una vez que alcancen la meta recibirán una recompensa, de tal manera que el esfuerzo realizado ha valido la pena.

En el modelo de fijación de metas $\mathrm{u}$ objetivos se considera que una meta $u$ objetivo es aquello que la persona intenta alcanzar (Trechera, 2005). Los factores que debe contemplar un objetivo para que motive son: el conocimiento, se debe conocer la meta y los medios requeridos para alcanzarla; la aceptación, debe existir acuerdo sobre lo que se desea realizar; la dificultad, las metas deben ser difíciles, pero no imposibles, y la especificidad, cuanto más concreto sea el objetivo, más fácil será aportar el esfuerzo para lograrlo.

\section{Ámbito educativo}

Una de las propuestas que mejor plantea la complejidad de los procesos motivacionales académicos, según Cerezo y Casanova (2007), es la que presentan Pintrich y De Groot, que distinguen tres categorías relevantes para la motivación en ambientes educativos: la primera se relaciona con uncomponente de expectativas, que incluye las creencias de las personas estudiantes sobre su capacidad para ejecutar una tarea; la segunda se asocia a un componente de valor, relacionado con sus metas y sus percepciones sobre la importancia e interés de la tarea; la tercera a un componente afectivo, que incluye las consecuencias afectivo-emocionales derivadas de la realización de una tarea, 
así como de los resultados de éxito o fracaso académico. Estos autores agregan que las investigaciones revelan que la persona se motiva más por el proceso de aprendizaje cuando confía en sus capacidades y posee altas expectativas de autoeficacia, además de valorar las actividades educativas $\mathrm{y}$ de responsabilizarse por los objetivos de aprendizaje.

Alonso (1992) se refiere a dos problemas motivacional-afectivos que enfrentan con frecuencia algunas personas estudiantes, que pueden atribuirse a las condiciones poco favorables en la institución educativa y al uso incorrecto de la dimensión afectiva por parte del personal docente. Estos problemas se refieren a la denominada indefensión y a la desesperanza aprendida. Estudiantes con el comportamiento de indefensión atribuyen el éxito académico a causas externas, cambiantes y fuera de su control. Señalan autocogniciones negativas y manifiestan que su inteligencia, memoria o su capacidad para resolver problemas son deficientes. Experimentan aburrimiento o ansiedad frente a las actividades educativas y a medida que aumentan las situaciones en que fracasan, disminuye su participación académica.

La desesperanza aprendida se puede observar en estudiantes que, aún si obtienen logros académicos, no les importa y piensan que están destinados a fracasar. Este tipo de estudiantes, que manifiestan indefensión y desesperanza aprendida, tienden a ver sus propias dificultades como fracasos insuperables, debido a sus limitadas habilidades.

\section{Teoría de las atribuciones y desempeño académico}

Bisquerra (2000) explica que en la Teoría de la Atribución de Heider se propone que las personas realizan atribuciones de causalidad que tienen efectos sobre las emociones, el pensamiento, la motivación, el comportamiento y la autoestima. En esta teoría las emociones se consideran como el resultado de atribuciones causales que inciden sobre las expectativas de éxito $y$, por ende, en la motivación que activa el comportamiento. De este modo, las expectativas altas facilitan la motivación y las bajas la desmotivación.

Pueden distinguirse cuatro tipos de estilo atributivo, que son: externalista: tanto los éxitos como los fracasos se atribuyen a causas externas (ambiente, otros); internalista: tanto los éxitos como los fracasos se atribuyen a causas internas de la persona (capacidad, esfuerzo); egoísta: los éxitos se atribuyen a causas internas y los fracasos a causas externas ("he aprobado", "me han suspendido"); y depresivo: los éxitos se atribuyen a causas externas y los fracasos a factores internos (Bisquerra, 2000).

\section{Motivaciones intrínseca y extrínseca en estudiantes}

Ajello (2003) señala que la motivación intrínseca se refiere a aquellas situaciones donde la persona realiza actividades por el gusto de hacerlas, independientemente de si obtiene un reconocimiento o no. La motivación extrínseca, por su parte, obedece a situaciones donde la persona se implica en actividades principalmente con fines instrumentales o por motivos externos a la actividad misma, como podría ser obtener una recompensa.

La motivación interna y el interés intrínseco en las actividades académicas aumentan cuando la persona tiene posibilidades deelección yoportunidades para tomar la responsabilidad de su aprendizaje, establecer sus propias metas, planear cómo alcanzarlas y monitorear su progreso. Por otra parte, cuando sus habilidades son altas pero las actividades no son desafiantes, el resultado es el aburrimiento. Cuando el desafío y los niveles de habilidad son bajos, se experimenta apatía y cuando se enfrenta una tarea desafiante para la que no se cree tener las habilidades necesarias, se experimenta ansiedad. 


\section{Autoestima}

De acuerdo con Rice (2000), la autoestima de una persona es la consideración que tiene hacia sí misma, es el ingrediente que proporciona dignidad a la existencia humana.

Por otra parte, de Mézerville (2004) menciona: "La autoestima es la percepción valorativa y confiada de sí misma, que motiva a la persona a manejarse con propiedad, manifestarse con autonomía y proyectarse satisfactoriamente en la vida”. (p. 61)

\section{Relación entre autoconcepto y autoestima}

Rice (2000) explica que todas las personas tienen seis aspectos del yo: lo que realmente son, lo que piensan que son, lo que piensan otras que son, lo que creen que piensan las demás de ellas, lo que quieren llegar a ser y las expectativas que creen que otras personas tienen sobre ellas. Los autoconceptos pueden o no tener enfoques cercanos a la realidad y están permanentemente en proceso de cambio.

Los estudios han demostrado una relación persistente entre el autoconcepto y la autoestima y diversas variables relacionadas con las actividades académicas. Por ejemplo, estudiantes con autoconceptos claros y una autoestima positiva tienden a participar más, a tener puntajes de competencia educativa más altas, a exhibir una conducta pro-social y a demostrar un logro académico mayor que el que tienen sus iguales con autopercepciones no claras o negativas.

\section{Componentes de la autoestima}

De acuerdo con de Mézerville (2004) existen una serie de aspectos interrelacionados que componen la autoestima. A partir de esta concepción, presenta un modelo propio al que ha denominado proceso de la autoestima. En este, se la considera según dos dimensiones complementarias; por una parte, una actitudinal inferida integrada por tres componentes: la autoimagen, la autovaloración y la autoconfianza; por otra parte, una dimensión conductual observable conformada por otros tres componentes: el autocontrol, la autoafirmación y la autorrealización.

La autoimagen consiste en la capacidad de verse a sí misma como la persona que realmente es, con sus virtudes y defectos. Generalmente los problemas de autoestima se asocian con una baja autoimagen; no obstante, también es posible que una autopercepción de superioridad señale dificultades en este aspecto, dando como resultado lo que se denomina pseudoautoestima.

La autovaloración consiste en que la persona se considere importante para sí misma y para las demás. Se relaciona con otros aspectos como la autoaceptación y el autorespeto y significa que percibe con agrado la imagen que la persona tiene de sí.

La autoconfianza se traduce en que la persona crea en sí misma y en sus propias capacidades para enfrentar distintos retos, lo que a su vez la motiva a buscar oportunidades que le permitan poner en práctica y demostrar sus competencias.

El autocontrol implica la capacidad de ordenarse apropiadamente y ejercer un dominio propio que fomente tanto el bienestar personal como el del grupo al que se pertenece. Por lo tanto, este término incluye otros aspectos, tales como el autocuidado, la autodisciplina, la organización propia y el manejo de sí.

La autoafirmación se define como la libertad de ser una o uno mismo y poder tomar decisiones para conducirse con autonomía y madurez. Se caracteriza por la capacidad que tiene la persona de manifestarse abiertamente cuando expresa sus pensamientos, deseos o habilidades. Además, incluye otras capacidades tales como la autodirección y la asertividad personal. 
La autorrealización consiste en el desarrollo y la expresión adecuada de las capacidades, de modo que la persona pueda vivir una vida satisfactoria y de provecho para sí misma y para otras personas. Implica la búsqueda del cumplimiento de las metas que conforman el proyecto vital de su existencia.

\section{Alta y baja autoestima}

El resultado de ser una persona con una alta autoestima y responsable de su propia vida es ser una persona actualizadora de sí misma y autotrascendente.

García (2008) señala que la persona con una alta autoestima cree firmemente en ciertos valores y principios, está dispuesta a defenderlos aún cuando encuentre fuertes oposiciones y se siente lo suficientemente segura como para modificarlos si nuevas experiencias indican que estaba equivocada. Es capaz de obrar según lo que crea más acertado confiando en su propio juicio. No emplea demasiado tiempo preocupándose por lo que haya ocurrido en el pasado, ni por lo que pueda ocurrir en el futuro. Tiene confianza en su capacidad para resolver sus propios problemas, sin dejarse acobardar por los fracasos y dificultades que experimente.

Además, la persona con alta autoestima se considera igual, como persona, a cualquier otra, aunque reconoce diferencias en talentos específicos, prestigio profesional o posición económica. No se deja manipular por los demás, aunque está dispuesta a colaborar si le parece apropiado. Reconoce y acepta en sí misma una variedad de sentimientos e inclinaciones tanto positivas como negativas. Es capaz de disfrutar diversas actividades. Es sensible a las necesidades de otras personas y respeta las normas de convivencia aceptadas.

De acuerdo con Rice (2000), las personas con baja autoestima muestran una identidad cambiante, inestable y vulnerable a la crítica o al rechazo. Puede que se perturben cuando sienten que las acusan o cuando perciben que otras personas tienen una opinión negativa de ellas. Cuanta más vulnerabilidad experimentan, mayores son sus niveles de ansiedad; como resultado, se sienten torpes e intranquilas y evitan a toda costa exponerse al ridículo. La baja autoestima de estas personas proviene de sus experiencias de vida, que probablemente le impidieron tener confianza en sí misma y funcionar de manera autónoma.

Las personas con baja autoestima, indican Molina, Baldares y Maya (1996), presentan características emocionales tales comoagresividad, timidez, alarde,impaciencia, competitividad, arrogancia, crítica, rebeldía ante figuras de autoridad y perfeccionismo, entre otras, las cuales propician conflictos en las relaciones interpersonales.

\section{La influencia de otras personas significativas en la autoestima}

Las agencias y los agentes del proceso de socialización desempeñan un importante papel en el desarrollo de la autoestima, ya sea positiva o negativamente. Las agencias de socialización incluyen a la familia, las instituciones educativas y los medios de comunicación. Los agentes socializantes son aquellas personas que moldean la conducta de la persona en su proceso de desarrollo, de modo que se ajuste a los requerimientos sociales. Así por ejemplo, los padres, madres, hermanas y hermanos, docentes e iguales son agentes de socialización, pero a la vez también son agentes de control social, pues controlan la conducta de la persona mediante amenazas, desaprobación o crítica; o bien, mediante la aprobación, el elogio o la recompensa. De esta manera se va definiendo en la persona su identidad, su autoconcepto y la valoración que hace de sí misma (Rice, 2000).

\section{Distorsiones cognitivas}

Beck (2002) y Ellis (2000) señalan que las situaciones no determinan los sentimientos, sino el modo en que las 
personas las interpretan; es decir, la respuesta emocional está condicionada por la percepción de la circunstancia.

De acuerdo con lo mencionado, se puede decir que el verdadero origen de la autoestima está en el interior de la persona. Depende de sus propias ideas, pero muchas de ellas pueden ser distorsiones cognitivas. Si se tuviera que definirlas de un modo más formal, indican Güell y Muñoz (2000), se podría decir que: "las distorsiones cognitivas son hábitos de pensamiento que habitualmente utilizamos para deformar negativamente la realidad". (p. 120)

Las evaluaciones negativas hechas por el sistema cognitivo provocan en los sistemas conductual y afectivo conductas y sentimientos negativos, acordes con la interpretación realizada. Se presentan entonces conductas como la huída, la evitación o el dejar de realizar actividades, emociones como apatía, tristeza y temor, todo lo cual repercute en la autoestima provocando que esta disminuya.

\section{Autoestima y resultados educativos}

Estudios realizados sobre la relación de distintas variables con el rendimiento académico muestran que las personas estudiantes con autoestima positiva, altas expectativas y motivación intrínseca para aprender, obtienen mejores logros académicos que aquellas con autoestima baja, expectativas escasas y una motivación extrínseca para el estudio.

Estas ideas son apoyadas por Rice (2000), quien explica que hay una correlación entre el autoconcepto y el rendimiento educativo. Las personas estudiantes con éxito tienen un mayor sentido de valía personal y se sienten mejor consigo mismas. Quienes tienen una autoestima alta tienden a tener mayor rendimiento académico, y quienes desarrollan su potencial académico tienen una autoestima más alta.

\section{Comunicación asertiva}

La palabra asertivo, de aserto, proviene del latín asertus y significa afirmación de la certeza de una cosa, por lo que se deduce que una persona asertiva es aquella que afirma con certeza.

Se considera que la asertividad es una conducta y no una característica de la personalidad, por lo que se habla de asertividad como una habilidad en el campo de las habilidades sociales.

Riso (2002) explica que una persona es asertiva cuando tiene la capacidad de ejercer o defender sus derechos personales; por ejemplo, decir "no", expresar opiniones contrarias o desacuerdos y manifestar sentimientos negativos sin permitir que la manipulen -como lo hace la persona sumisa-, pero tampoco violando los derechos de otras personas, como lo hace la agresiva.

De acuerdo con Melgosa (1995), la asertividad consiste en "la expresión de nuestros sentimientos de una manera sincera, abierta y espontánea, sin herir la sensibilidad de la otra persona”. (p. 84)

\section{Características de la asertividad}

En opinión de Güell y Muñoz (2000), la persona asertiva evita que la manipulen, es más libre en sus relaciones interpersonales, posee una autoestima más alta, tiene más capacidad de autocontrol emocional y muestra una conducta más respetuosa hacia las demás personas. Consideran estos autores que el modelo de conducta asertiva es difícil de llevar a la práctica, pues muchas veces durante el proceso de socialización se aprende la pasividad, pero, por el contrario, también el entorno social, los medios de comunicación y una educación competitiva fomentan la conducta agresiva. Por consiguiente, comportarse de una manera asertiva implica a veces conducirse de manera contraria a los 
modelos de conducta que se practican más frecuentemente.

Las conductas no asertivas son la conducta agresiva y la pasiva. Güell y Muñoz (2000) comentan, respecto de la conducta pasiva, que se ha comprobado que se encuentra relacionada con sentimientos de culpabilidad, ansiedad y sobre todo, con baja autoestima. Las personas pasivas siempre tienen temor de molestar a las demás, tienen dificultad para afrontar una negativa o un rechazo y sufren de un sentimiento de inferioridad. Por otra parte, en la conducta agresiva, las consecuencias a corto plazo pueden parecer favorables, por cuanto las personas consiguen de forma agresiva o violenta sus propósitos. No obstante, esta conducta anula el derecho de las otras personas y genera odio y resentimiento.

Otras formas de comportamiento no asertivo, de acuerdo con Neidhardt, Weinstein y Conry (1989), son la culpabilidad, la crítica, el ridículo y dudar de los motivos, comentadas a continuación.

La culpabilidad. La persona que utiliza la manipulación puede intentar provocar sentimientos de culpabilidad para forzar a otra a actuar de cierta manera.

La crítica. Otra forma de manipulación es criticar la conducta de otra persona. En estas situaciones, el propósito que se persigue con la crítica es poner a la otra persona a la defensiva, evitando que continúe centrando su atención en la persona que hace la crítica.

El ridículo. Con frecuencia se trata de una crítica no solicitada. Es probable que la persona intente desviar la atención que en ese momento está centrada sobre ella. Lo más corriente es que se utilice como una forma indirecta de persuadir, a base de avergonzar o poner en ridículo a otra persona para que realice algo que no desea hacer.

Dudar de los motivos. La persona que pregunta sobre los motivos de la conducta de otra probablemente quiera criticar su decisión o sus razones, pero no tiene el valor suficiente para expresar la crítica de forma directa.

\section{Creencias y pensamientos en las conductas asertivas y no asertivas}

Las personas no asertivas piensan, sienten y se comportan de una forma débil cuando deben ejercer o defender sus derechos. De acuerdo con Riso (2002), los pensamientos típicos que caracterizan a una persona no asertiva pueden resumirse en que los derechos de las demás son más importantes que los propios; ella no puede herir los sentimientos de las otras personas ni ofenderlas, aunque tenga la razón y la perjudique; piensa que si expresa sus opiniones, será criticada o rechazada y no sabrá qué decir ni cómo decirlo. Así mismo, no se siente hábil para expresar sus emociones.

En el caso de las personas agresivas, la creencia que rige su comportamiento es que sus derechos son más importantes que los de las demás. Su conducta infunde temor, pero no respeto.

Güell y Muñoz (2000) se refieren a las creencias y actitudes que caracterizan las conductas asertivas. Aquellas personas que son asertivas consideran que tanto ellas como las otras tienen derecho de intentar conseguir lo que consideren mejor, siempre que no repercuta negativamente sobre otras personas; además, el derecho de ser respetadas; asimismo, de solicitar ayuda, no de exigirla; también, de sentir emociones, y de expresarlas sin herir los sentimientos de las demás; igualmente, de tener sus propias opiniones sobre cualquier situación y a manifestarlas sin ofender a otras personas; $y$, al mismo tiempo, de equivocarse en sus actitudes, opiniones y comportamientos y a ser responsables de ello.

Uno de los propósitos fundamentales de la asertividad es mejorar la comunicación entre las personas y facilitar formas de llegar a acuerdos y a compromisos cuando las opiniones y los intereses son opuestos.

\section{Asertividad y adolescencia}

Muchos adolescentes no tienen dificultades especiales en sus comunicaciones. 
Son personas abiertas, francas y muy sociables, señalan Güell y Muñoz (2000). Pero también existen las personas adolescentes que tienen problemas de retraimiento, timidez, inseguridad, para quienes su dificultad de comunicación se convierte en un problema de personalidad más serio.

Evaluación y autoevaluación, observar y auto-observarse son dos procesos inseparables que definen toda relación social, indica Riso (2002). De estas dos operaciones mentales surge el modo en que la persona se relaciona con las demás. Si esta se siente segura de sí misma (evaluación del yo) y percibe a las otras personas significativas como amigables y no amenazadoras (evaluación de las otras) se sentirá cómoda, espontánea y tranquila frente a las demás. El temor a la evaluación negativa será mínimo o nulo. Si ocurre la situación inversa, la persona puede tener problemas de ansiedad social, lo cual implica resolver el dilema de querer y necesitar de la gente, pero sentir temor de lo que esta pueda pensar de su persona. Si esta se aleja, se deprime y si se acerca, el temor la inmoviliza.

Las cualidades personales y las habilidades sociales son criterios significativos de popularidad y se han considerado muy importantes para obtener aceptación social. Santrock (2002) expresa que las personas estudiantes que son más aceptadas por sus pares y que poseen buenas habilidades sociales, con frecuencia obtienen mejores promedios, manifiestan una actitud positiva y una mayor motivación de logro académico. En contraste, quienes son rechazadas, en especial las que manifiestan una conducta agresiva, están en riesgo de presentar problemas de rendimiento, con calificaciones bajas y deserción de la institución educativa.

\section{Estrés}

Todas las personas necesitan aprender a prevenir y controlar el estrés. Quien no lo hace, puede poner en peligro su salud y su tranquilidad, mientras que quien conoce y pone en práctica acciones adecuadas para prevenirlo y afrontarlo puede disfrutar de un estilo de vida más sano y más satisfactorio.

Autores como Oblitas (2004) se refieren a diferentes concepciones teóricas y cómo estas entienden el término estrés. Explica que los enfoques fisiológicos y bioquímicos se centran en las respuestas orgánicas que se generan en la persona cuando se enfrenta a una situación percibida como amenazante. Los enfoques psicosociales hacen énfasis en factores externos; es decir, en los estímulos y eventos productores de estrés. Los enfoques con una orientación cognitiva subrayan que el estrés surge a partir de la evaluación cognitiva que la persona realiza tomando en cuenta tanto aspectos internos como aquellos propios del ambiente. Finalmente, los enfoques más integradores consideran que el estrés debe comprenderse desde una perspectiva más amplia, por cuanto en este convergen e interactúan muchas variables.

\section{Eustrés, distrés y nivel óptimo de estrés}

El buen estrés, esto es, eustrés, es todo aquello que causa placer, todo lo que la persona quiere o acepta hacer en armonía consigo misma, con su medio y con su propia capacidad de adaptación. Es el estrés de la realización agradable. Ejemplos de buen estrés son la alegría, el éxito, el afecto, el trabajo creador, un rato de tranquilidad, compartir con otras personas, aspectos que resultan estimulantes, alentadores, fuente de bienestar, de felicidad o de equilibrio.

El mal estrés o distrés es todo aquello que disgusta, todo cuanto la persona hace a pesar suyo, en contradicción consigo misma, su ambiente y su propia capacidad de adaptación. La tristeza, el fracaso, las malas noticias, la enfermedad, las presiones, la carencia de libertad, las frustraciones, son ejemplos de mal estrés, 
fuentes de desequilibrio, de alteraciones psicosomáticas y de enfermedades de adaptación.

A cada persona le conviene conocer su nivel de estrés, sus límites y capacidades de adaptación así como la dosis de presión que puede soportar, para evitar situarse en condiciones que superen su umbral de tolerancia al estrés.

\section{Fases del estrés}

Desde que aparece hasta que alcanza su máximo efecto, el estrés pasa por tres etapas: alarma, resistencia y agotamiento (Melgosa, 1995).

La fase de alarma, constituye el aviso claro de la presencia de un agente estresante. Las reacciones fisiológicas ante este agente son las primeras que aparecen, para advertir a la persona que debe ponerse en estado de alerta.

Cuando el estrés prolonga su presencia más allá de la fase de alarma, la persona entra en la segunda fase, denominada de resistencia. Intenta continuar enfrentado la situación, pero se da cuenta de que su capacidad tiene un límite, se frustra y sufre como consecuencia de esta situación.

Posteriormente se presenta la fase de agotamiento que es la fase terminal del estrés. Se caracteriza por la fatiga, la ansiedad y la depresión, que pueden aparecer por separado o simultáneamente. La fatiga generalmente va acompañada de nerviosismo, irritabilidad, tensión e ira.

\section{La respuesta al estrés}

Además de las respuestas físicas, tales como pulso acelerado, aumento de la sudoración, contracción del estómago, tensión en los músculos y respiración rápida y entrecortada, existen otras respuestas ante el estrés. Neidhardt et al. (1989) las categorizan en dos grupos, las psíquicas y las de conducta. Respecto de las respuestas psíquicas, las más generalizadas son: incapacidad para concentrarse; dificultad para tomar decisiones, incluso las más sencillas; pérdida dela confianza en sí misma o en sí mismo; irritabilidad; preocupaciones y ansiedad; y temores irracionales o pánico sobrecogedor.

En cuanto a las respuestas de conducta, se mencionan algunas como: aumento en el empleo de medicamentos, tics nerviosos, distracción, proclividad a los accidentes, comer en exceso o falta de apetito, dormir demasiado o insomnio, aumento en el consumo de alcohol o drogas, impulsividad y agresividad.

Un enfrentamiento inadecuado del estrés llega hasta la aparición de síntomas y trastornos psicosomáticos (Bensabat, 1987). Sobre este tema Melgosa (1995) explica que: "se entiende por trastornos, reacciones o enfermedades psicosomáticas, aquellas dolencias que dan manifestaciones orgánicas, pero tienen su origen en causas psicológicas, como el propio estrés u otros estados mentales adversos". (p. 44)

\section{Causas del estrés}

En general, se puede decir que existen dos fuentes fundamentales del estrés. En primer lugar, están los agentes estresantes que provienen de circunstancias externas, del ambiente, de la familia, del trabajo, del estudio, entre otras. En segundo lugar, se encuentra el estrés producido por la persona misma, es decir, por sus propias características de personalidad y por su forma particular de enfrentar y solucionar los problemas.

Diferentes autores asocian los distintos tipos de afrontamiento al estrés de acuerdo con las diferencias en los rasgos de personalidad. Melgosa (1995) menciona la distinción entre tipos de personalidades, a las que se denominan el tipo A, el tipo B y un tipo C. Por supuesto, existe una gama de tipos intermedios con características de los distintos tipos. 
El tipo A es extrovertido, corresponde a las personas que exteriorizan su respuesta al estrés mediante reacciones excesivas. La menor contrariedad se traduce en irritabilidad y accesos de cólera desproporcionados en relación con los factores estresantes. Estas personas son impacientes crónicas, se empujan a sí mismas y a las demás, tienen dificultades para relajarse y se enfrascan en sus trabajos sacrificando muchas veces otras dimensiones de su vida, como la familiar y la afectiva.

La persona con el tipo de personalidad C, por el contrario, es introvertida y obsesiva. Interioriza su reacción al estrés. Aparentemente son resignadas y apacibles, pero se trata de falsas calmas, puesto que sufren en silencio.

El tipo de personalidad B es el intermedio, el modo ideal de comportamiento. Corresponde a la persona que domina de forma adecuada y natural su estrés, reacciona con calma, con prudencia y con una buena perspectiva de la situación. Su carácter es optimista. Este tipo de personalidad es minoritario, mientras que los tipos $\mathrm{A}$ y $\mathrm{C}$ son más frecuentes entre la población.

Otras posibles causas de estrés a las que hace referencia Melgosa (1995) son las experiencias traumáticas, las molestias cotidianas, el ambiente físico y social, las elecciones conflictivas y algunas circunstancias estresantes típicas que se presentan a lo largo de la vida.

\section{Prevención del estrés}

Para poder prevenir y afrontar el estrés de manera óptima el primer requisito es que la persona se fortalezca física y psicológicamente, de manera que pueda hacer frente y resistir a las inevitables circunstancias de la vida.

Otros aspectos a los que se debe prestar atención para prevenir el estrés, de acuerdo con autores tales como Bensabat (1987), Neidhardt et al. (1989) y Melgosa (1995), son, por ejemplo: el ejercicio físico y el reposo, la buena alimentación, el contacto con la naturaleza, el tiempo libre, la planificación del tiempo, de objetivos y actividades.

En cuanto al ejercicio y al reposo, se menciona que la mayoría de las personas que sufren de estrés no practican ningún ejercicio físico, ni son capaces de lograr un descanso adecuado, lo cual genera un círculo vicioso de difícil salida. Sobre el descanso, este debe ser cotidiano, semanal, anual y además debe ser de calidad, de modo que permita recuperarse del desgaste tanto físico como psíquico que conlleva la realización de las actividades. Los buenos hábitos relacionados con la alimentación son importantes siempre, pero sobre todo cuando hay estrés, esto por cuanto, si la persona está sufriendo de estrés tiende a comer en exceso, a no comer lo necesario o hacerlo deprisa y en cualquier momento.

El contacto con la naturaleza es una experiencia relajante integral. La persona obtiene en la interacción con esta paz y salud. Utilizar de forma adecuada el tiempo libre compensa los efectos negativos del exceso de trabajo o de estudio, ayuda a afianzar las relaciones familiares y sociales y permite cambiar el ritmo y dejar de lado los problemas cotidianos. La planificación personal también ayuda a prevenir el estrés. Se sugieren dos pasos: primero, identificar los objetivos personales; segundo, organizar el tiempo disponible de la mejor manera, elaborando un programa realista de actividades que permita cumplir con estas sin un exceso de presión.

\section{Afrontar el estrés}

El estrés puede ser controlado. La persona puede adiestrarse para desarrollar determinadas habilidades que le ayuden a identificar factores potenciales de estrés y a modificar reacciones perjudiciales ante él.

Una de las habilidades que se pueden desarrollar es la de lograr relajarse. Además del beneficio inmediato que brinda, 
otorga a la persona la sensación de estar en control de sí misma.

Otra forma de controlar el estrés es aprender a preocuparse constructivamente. De acuerdo con Melgosa (1995), existen dos tipos de preocupación, la destructiva y la constructiva. La preocupación destructiva es aquella que no está provista de estrategias de solución, es repetitiva y obsesiva. En cuanto a la preocupación constructiva, es racional, incluye planes y métodos para afrontar los agentes productores de estrés $\mathrm{y}$ se centra fundamentalmente en las posibles soluciónes.

Naranjo (2004) describe una serie de métodos mediante los cuales la persona puede lograr un mejor control del estrés, estos son: establecer las situaciones causantes del estrés; medir el nivel de estrés; disputar los pensamientos irracionales; practicar la relajación física y mental; fomentar la autoestima; comunicarse asertivamente y tratar de modificar el tipo A de personalidad, por cuanto corresponde a un estilo de comportamiento que frecuentemente despierta la respuesta de estrés.

\section{Estrés en la educación}

Señala Rice (2000) que cualquier situación que perturbe la armonía e interfiera en las actividades de la clase puede ser fuente de estrés y afecta el funcionamiento académico en el acontecer educativo.

Dumont, LeClerc y Deslandes (2003) encontraron que un alto porcentaje de estudiantes evidencian síntomas de ansiedad antes de presentar una prueba. Los niveles altos de ansiedad pueden ser también el resultado de las expectativas irreales y la presión de las madres y de los padres respecto del logro educativo de sus hijas e hijos. Por su parte, Jadue (2001) señala que el fracaso es fuente de frustración en una institución educativa que estimula la competitividad en sus estudiantes. La frustración es uno de los factores que puede llevar a una conducta agresiva, ya que fomenta la hostilidad entre estudiantes, de modo que cuando compiten por rendimiento académico la violencia dentro de la escuela aumenta.

Newcomer (1993) menciona que los altos niveles de estrés y ansiedad reducen la eficiencia en el aprendizaje, ya que disminuyen la atención, la concentración y la retención, con el consecuente deterioro en el rendimiento educativo. A medida que va procesando la información, el estudiantado ansioso no logra organizar ni elaborar adecuadamente los contenidos de las materias y tiende a ser poco flexible para adaptarse a los procesos de aprendizaje. Martínez y Díaz (2007) señalan que: "El estrés escolar se dimensiona en el desequilibrio psíquico, afectivo, cognitivo y social del estudiante”. (p. 20)

\section{Metodología}

\section{Tipo de investigación}

Esta investigación es de tipo descriptivo. Pretende conocer y describir cuáles son los factores personales que favorecen el desarrollo de una actitud positiva hacia las actividades académicas, de acuerdo con la opinión de un grupo de estudiantes de décimo año.

De acuerdo con Hernández (1998), la investigación descriptiva consiste en registrar e interpretar hechos presentes que permitan alcanzar una meta significativa. En el caso de esta investigación, se pretende obtener conclusiones que brinden un aporte a la educación para que desde esta se favorezcan aquellos factores que coadyuvan en el desarrollo de actitudes positivas del estudiantado hacia las actividades académicas.

\section{Población}

La población, según Barrantes (2004), está conformada por "un conjunto de elementos que poseen una característica 
en común” (p. 135). La población de esta investigación, realizada en el año 2007, está integrada por todo el estudiantado de educación secundaria que cursa el décimo año. La muestra está conformada por cincuenta estudiantes de décimo año que asisten a las siguientes instituciones educativas: Liceo de San Antonio de Desamparados y Colegio de Santa Ana. Esta muestra es intencional, de acuerdo con lo que explica Gómez (2005, p. 10): “o sea utilizando el juicio de una persona con experiencia y conocimiento con respecto a la población que se estudia”.

\section{Conceptos y definiciones}

Esta investigación está constituida por un constructo principal, esto es, la actitud positiva hacia las actividades académicas, y un subconstructo: los factores personales que favorecen esas actitudes. Los aspectos que integran este subconstructo son la autoestima positiva, la motivación, la comunicación asertiva y el manejo de estrés.

\section{Actitudes positivas hacia las actividades académicas}

La actitud es una fuerza interna, una creencia o un sentimiento de una persona, que determina su predisposición a valorar positivamente los objetos y sujetos que la rodean y que, por lo tanto, influye en su comportamiento (Océano, 1998).

\section{Factores que favorecen el desarrollo de actitudes positivas hacia las actividades académicas}

Para efectos de esta investigación, se entenderá como aquellos aspectos propios de cada estudiante que favorecen su disposición hacia las actividades académicas, entre ellos la autoestima positiva, la motivación, la comunicación asertiva y el manejo del estrés.

\section{Autoestima positiva}

Es el valor que se atribuye una persona y que le permite experimentar sentimientos y pensamientos positivos respecto de sí misma.

\section{Motivación}

Es una fuerza interna que impulsa a la persona a actuar o realizar algo, que le permite reflejar el interés en distintos niveles de su desempeño como persona.

\section{Comunicación asertiva}

Consiste en la "expresión de nuestros sentimientos y pensamientos de una manera sincera, abierta y espontánea, sin herir la sensibilidad de otra persona" (Melgosa, 1995, p. 84).

\section{Manejo del estrés}

La capacidad que tiene la persona de enfrentar de forma adecuada el estrés que pueden provocarle situaciones del diario vivir.

\section{Recolección de información}

El instrumento que se utiliza en esta investigación para recolectar la información es el cuestionario, entendido como el conjunto de afirmaciones respecto de conceptos y definiciones (Hernández, 1998). Este tipo de instrumento, de acuerdo con Gómez (2005), permite obtener información pertinente y confiable mediante la formulación de afirmaciones adecuadas para cumplir con los objetivos propuestos. 
El cuestionario que se utiliza en esta investigación consta de dos partes. La primera hace referencia a indicaciones de tipo general y la segunda incluye una serie de afirmaciones específicas relacionadas con el tema de estudio.

Las afirmaciones que se utilizan contienen categorías u opciones de respuestas, o sea, se le presenta a la persona las posibilidades de respuestas y ella debe circunscribirse a éstas (Barrantes, 2004).

Para responder a las afirmaciones se emplea una escala de Likert, la cual consiste en una serie de opciones de entre las cuales la persona debe seleccionar una, aquella que, de acuerdo con su criterio, considere más adecuada para responder a la afirmación que se le presenta. Las opciones de esta escala son: muy de acuerdo, de acuerdo, ni de acuerdo ni en desacuerdo, en desacuerdo y muy en desacuerdo.

\section{Instrumentalización}

El cuestionario está constituido por cuarenta afirmaciones, diez afirmaciones por cada indicador del subconstructo factores personales, que confirman el constructo principal, actitudes positivas hacia las actividades académicas.

Cuadro 1

\section{Instrumentalización}

\begin{tabular}{|c|c|c|}
\hline Constructo & Subconstructo & Indicadores \\
\hline $\begin{array}{l}\text { Actitudes positivas } \\
\text { hacia las actividades } \\
\text { académicas. }\end{array}$ & $\begin{array}{l}\text { Factores personales que favorecen } \\
\text { el desarrollo de actitudes positivas } \\
\text { hacia las actividades académicas. }\end{array}$ & $\begin{array}{ll}\text { - } & \text { Motivación } \\
\text { - } & \text { Comunicación asertiva } \\
\text { - } & \text { Autoestima positiva } \\
\text { - } & \text { Manejo del estrés }\end{array}$ \\
\hline
\end{tabular}

\section{Procesamiento de los datos}

De acuerdo con la naturaleza de esta investigación, se utiliza la estadística descriptiva, la cual se refiere al empleo de técnicas que se usan cuando se desea describir un conjunto de datos (Gómez, 2005).

\section{Análisis de los resultados}

La motivación se considera un aspecto de enorme relevancia por cuanto es una fuerza que mantiene el curso de las acciones de la persona y determina, en gran medida, su nivel de rendimiento. Se le considera, en el ámbito educativo, como una disposición o actitud positiva para aprender y seguir haciéndolo de forma autónoma.
De acuerdo con los resultados, la población estudiantil concede importancia tanto a factores internos como externos relacionados con la motivación, como significativos para el desarrollo de actitudes positivas hacia las actividades académicas. De esta manera, por un lado señalan como importantes factores internos tales como: sentir seguridad en sus capacidades (100\%), participar en actividades que para la persona son significativas e importantes (90\%), plantearse objetivos y metas que se desea alcanzar (88\%) y asumir la responsabilidad de sus logros académicos (88\%). Por otro lado, factores externos de la motivación, tales como: sentir seguridad, afecto y atención de otras personas que consideran importantes (90\%) y obtener buenas calificaciones en pruebas y trabajos (88\%). 
En el caso de los factores externos, las personas se implican en actividades, tales como el estudio, por motivos ajenos a las actividades mismas, mediante las cuales obtienen una recompensa, en este caso, lograr buenas calificaciones. Esta posición es opuesta a la motivación intrínseca, en la que las personas realizan actividades de aprendizaje únicamente por el gusto de hacerlas, e independientemente de obtener reconocimiento. La población estudiantil consideró, al plantearle la afirmación anterior, que no está ni de acuerdo ni en desacuerdo con ella (42\%) y en desacuerdo con la afirmación (26\%).

De acuerdo con Harter (citado por Universidad de Sevilla, 2005), los estudios han demostrado que la motivación intrínseca decae a lo largo de los años de colegio, aspecto que se atribuye a que el estudiantado percibe un mayor énfasis por parte del personal docente en las notas, realzando de esta forma la evaluación externa del desempeño. Esa necesidad de reconocimiento posiblemente se asocia con el alto porcentaje de estudiantes (90\%) que está de acuerdo y muy de acuerdo con la afirmación: "siento seguridad, afecto y atención de otras personas que son importantes para mí", recompensa que quizá obtengan logrando buenos resultados en pruebas y trabajos.

No obstante, como se mencionó, esta población de adolescentes considera también relevantes factores internos de la motivación; por ejemplo, sentir seguridad en sus capacidades, sobre lo cual menciona Ajello (2003) que la opinión que sobre sí tenga la persona respecto de sus propias habilidades determina el tipo y duración del esfuerzo que esta realiza y, por lo tanto, el resultado de sus acciones.

Sobre el aspecto de participar en actividades significativas e importantes, un 90\% del estudiantado señala estar de acuerdo y muy de acuerdo en que esto favorece sus actitudes positivas hacia las actividades académicas. Harter (citado por Universidad de Sevilla, 2005) menciona que cuando las personas sienten que el trabajo académico es irrelevante y aburrido tienden a perder su motivación. Por otra parte, la perspectiva humanística subraya la capacidad del ser humano para lograr su crecimiento personal, elegir su destino y las cualidades positivas, lo cual se confirma en los altos porcentajes obtenidos (88\%) del estudiantado, que considera como importantes los aspectos: "plantearme objetivos y metas que deseo alcanzar y asumir la responsabilidad de mis logros académicos".

La autoestima es otro factor de relevancia en el desarrollo de actitudes positivas hacia las actividades académicas. La autoestima es la consideración que la persona tiene hacia sí misma y refleja la confianza global de esta y su satisfacción personal. Se refiere al valor del yo y debería ser el propósito más alto del proceso educativo.

De acuerdo con Rice (2000), cuando las personas estudiantes, mediante su autoconocimiento, pueden autoaceptarse y aprobarse, esto las conduce a un sentimiento de valía que les permite tener la autoestima suficiente para aceptarse a sí mismas. Esta afirmación es corroborada por el estudiantado, que estima importante, en un alto porcentaje (96\%), considerarse personas valiosas aunque no sean perfectas. Al respecto señala Rogers (1998) que su posición ante la vida parece ser la de que gustan de sí mismas aún cuando saben que no son personas perfectas.

Esta población estudiantil también considera de enorme importancia el aspecto de la autovaloración, al señalar en un 100\% que siente que tiene el derecho de triunfar y de ser feliz. Branden (citado por de Mézerville, 2004) define la autovaloración como: "la confianza en nuestro derecho a triunfar y a ser felices, el sentimiento de ser respetables, de ser dignos". (p. 34)

García (2008) considera que una de las características de una persona con alta autoestima es la de tener confianza en su capacidad para resolver sus propios problemas, sin dejarse acobardar por los fracasos y dificultades que experimente, afirmación con la que concuerda un alto porcentaje de 
la población estudiantil (94\%) y la asocia con el desarrollo de actitudes positivas hacia las actividades académicas. Las creencias que sobre sí tengan las personas parecen actuar como poderosos determinantes de la conducta. Molina et al (1996) añaden que la opinión que se tiene de la propia persona y de su conducta ocupa un lugar importante en la consolidación de la autoestima.

En cuanto a la comunicación asertiva, es una habilidad social. Facilita que la persona se sienta más satisfecha consigo misma y con las demás, pues permite la expresión apropiada de los pensamientos y las emociones en las relaciones sociales, sin que se produzca ansiedad o agresividad.

De acuerdo con Güell y Muñoz (2000), uno de los componentes de tipo ético que se presupone en la actitud asertiva es que ninguna persona tiene derecho de aprovecharse de las demás. En la autoafirmación o asertividad se parte del concepto de igualdad entre las personas y del derecho de cada una de manifestar las opiniones propias. Implica respeto por las demás personas y por una misma, es decir, respeto por los derechos de los seres humanos y el derecho de defender sus propios derechos. El estudiantado concuerda con las afirmaciones anteriores, pues entre un $90 \%$ y un $92 \%$ señala estar de acuerdo y muy de acuerdo en sentirse capaces de respetar sus propios derechos y los de las otras personas y en sentirse igual a estas, ni superior ni inferior a ellas, y asocian estos factores como favorecedores del desarrollo de actitudes positivas hacia las actividades académicas.

Otro aspecto considerado importante por la población estudiantil (92\%) es sentirse capaz de no dejarse manipular y decir no cuando alguien intenta que haga algo que no desea. Ciertamente, la persona asertiva evita que la manipulen, es más libre en sus relaciones interpersonales y muestra una conducta más respetuosa hacia las demás personas. Riso (2002) reafirma la idea anterior al señalar que la persona es asertiva cuando puede decir que no, sin permitir que la manipulen, como lo hace la sumisa, y sin manipular, como lo hace la agresiva.

También un $84 \%$ de la población estudiantil señala como un factor importante el poder dar y recibir elogios, y expresar sentimientos positivos y de forma sincera. Al respecto, Riso (2002), al referirse a la conducta asertiva, señala que es "aquella conducta que permite a la persona expresar adecuadamente (...) oposición (...) y afecto (dar y recibir elogios, expresar sentimientos positivos en general)". (p. 45)

Neidhardt et al. (1989) señalan que asumir una conducta defensiva puede ser indicio de falta de confianza de la persona en sí misma, el cual podría ser el caso de un $26 \%$ a un $30 \%$ de la población estudiantil, quienes opinan no estar ni de acuerdo ni en desacuerdo con las afirmaciones de que poder considerar puntos de vista opuestos a los suyos como una oportunidad para aprender y poder expresar sus reclamaciones con cortesía y a la vez escuchar las reclamaciones de otras personas favorezcan actitudes positivas hacia las actividades académicas.

El estrés, entendido como el conjunto de reacciones fisiológicas y psicológicas que experimenta la persona ante una demanda, es un tema de interés en el estudio de la conducta humana, por cuanto sus efectos tienen repercusiones tanto en la salud física como mental. Por lo tanto, resulta de gran importancia que las personas aprendan a prevenir y afrontar el estrés de forma adecuada, pues ello les permitirá disfrutar de un estilo de vida más sano y satisfactorio.

De acuerdo con los resultados de esta investigación, el 96\% de la población estudiantil considera importante sentir entusiasmo por realizar sus actividades favoritas y un $90 \%$ estima también de importancia sentirse bien consigo misma y asocia ambos aspectos con el desarrollo de actitudes positivas hacia las actividades académicas. Al respecto, Melgosa (1995) señala que cuando las personas sufren de estrés y este se asocia con depresión, carecen de motivación para encontrar placenteras sus 
actividades y los sentimientos hacia sí son cada vez más negativos.

Cuando la persona se encuentra deprimida (lo cual muchas veces se vincula con el estrés), no realiza proyectos ni siente deseos de proponerse ninguno, el inicio de toda actividad resulta demasiado pesado y hay una pérdida de la estima de sí (Bensabat 1987). La autoestima y la buena disposición son ejemplos de rasgos de la personalidad que desempeñan un papel importante frente a las situaciones estresantes.

Otros factores que la población adolescente señala como importantes para el desarrollo de actitudes positivas hacia las actividades académicas son: poder relajarse, descansar y dormir profundamente, en un $86 \%$, y sentirse optimista, de buen ánimo y humor y sentir que sus condiciones físicas son óptimas, en un $82 \%$. Sobre el aspecto del descanso, Bensabat (1987) menciona que ciertamente, cuando el organismo se encuentra súper estimulado, agotado por cansancio, sometido a una adaptación excesiva, exige una secreción importante de hormonas de adaptación (adrenalina y cortisona), responsables de efectos secundarios y de alteraciones metabólicas y orgánicas.

Neidhardt et al. (1989) indican que se emplea el término eustrés para definir la situación en que la buena forma física y el bienestar mental facilitan que el cuerpo en su conjunto adquiera y desarrolle su máximo potencial. El estado de eustrés se asocia con claridad mental y condiciones físicas óptimas. Los autores mencionados, Bensabat (1987) y Melgosa (1995), asocian los aspectos anteriores con el ejercicio físico, señalando que este facilita el descanso, aumenta la capacidad y agilidad mental, mejora el estado de ánimo y favorece el buen humor y la autoestima, aspectos que también la población estudiantil considera importantes (82\%).

El descanso, poder relajarse y dormir profundamente permiten a la persona recuperarse del desgaste tanto físico como psíquico que conlleva la realización de actividades. Newcomer (1993) menciona que el estrés y la ansiedad reducen la eficacia en el aprendizaje, ya que disminuyen la atención, la concentración y la retención, con el consecuente deterioro en el rendimiento educativo. De allí la importancia, señala Santrock (2002), de que se desarrollen diversos programas de intervención destinados a reducir los niveles de tensión y ansiedad en la población estudiantil.

\section{Conclusiones}

A partir del análisis realizado, resulta evidente que la autoestima positiva, la motivación, la comunicación asertiva y el manejo del estrés favorecen el desarrollo de una actitud positiva de la población estudiantil hacia las actividades académicas.

De acuerdo con los objetivos específicos planteados en esta investigación, se puede concluir además, según la opinión de dos grupos de estudiantes de décimo año, que:

Los aspectos más importantes relacionados con la autoestima que favorecen el desarrollo de actitudes positivas hacia las actividades académicas son sentir que tienen derecho de triunfar y ser felices, poder considerarse una persona valiosa aunque no sea perfecta y sentir que tienen la capacidad para resolver sus propios problemas sin dejarse vencer por fracasos o dificultades.

Otros factores que obtuvieron porcentajes elevados, entre un $86 \%$ y un $92 \%$, son sentir orgullo de sus logros, pero también poder aceptar sus limitaciones; ser capaz de tomar decisiones y conducirse con autonomía; sentir que es importante para sí misma y para otras personas; pensar que puede realizar bien distintas cosas y sentir seguridad al hacerlas y sentirse capaz de relacionarse adecuadamente con otras personas.

Los aspectos más importantes relacionados con la motivación que favorecen el desarrollo de una actitud positiva hacia las actividades académicas son sentirse segura (o) de sus capacidades; 
sentir seguridad, afecto y atención de otras personas que consideran importantes para sí; participar en actividades que son significativas o importantes; plantearse objetivos y metas que desean alcanzar; asumir la responsabilidad de sus logros académicos y obtener buenas calificaciones en pruebas y trabajos.

Otros dos aspectos que también obtuvieron porcentajes altos, un $84 \%$ y $82 \%$ respectivamente, son poder poner en práctica sus habilidades y sentirse segura (o) de que se va a obtener un éxito académico.

Los aspectos más importantes relacionados con la comunicación asertiva que favorecen el desarrollo de una actitud positiva hacia las actividades académicas son sentirse capaz de no dejarse manipular y decir no cuando alguien intenta que haga algo que no desea; sentirse capaz de respetar sus derechos y los derechos de otras personas; sentirse igual a las otras personas, ni superior ni inferior a ellas $\mathrm{y}$ poder dar y recibir elogios y expresar sentimientos positivos y de forma sincera.

Otros aspectos que también obtuvieron porcentajes altos, entre un $76 \%$ y un $82 \%$, son poder defender sus derechos sin temor y sin agresión; sentirse capaz de hacer y recibir críticas constructivas y poder expresarse adecuadamente y sin ansiedad.

Los aspectos más importantes relacionados con el manejo del estrés que favorecen el desarrollo de actitudes positivas hacia las actividades académicas para las personas estudiantes son sentir entusiasmo por realizar sus actividades favoritas, sentirse bien consigo mismas y poder relajarse, descansar y dormir profundamente.

Otros dos factores que también obtuvieron un porcentaje alto (82\%) son sentirse optimista, de buen ánimo o humor y sentir que sus condiciones físicas son óptimas.

\section{Referencias bibliográficas}

Ajello, A. M. (2003). La motivación para aprender. En C. Pontecorvo (Coord.).
Manual de psicología de la educación (pp. 251-271). España: Editorial Popular.

Alonso, J. (1992). ¿Qué es lo mejor para motivar a mis alumnos: análisis de lo que los profesores saben, creen y hacen al respecto? Madrid: Universidad Autónoma. Instituto de Ciencias de la Educación.

Bandura, A. (1994). Self-efficacy: The exercise of control. New York: W.H. Freeman.

Barrantes, R. (2004). Investigación: Un camino al conocimiento un enfoque cualitativo y cuantitativo. San José: EUNED.

Beck, J. (2002). Terapia cognitiva. Conceptos básicos y profundización. Barcelona: Gedisa.

Bensabat, S. (1987). Stress. Grandes especialistas responden. Bilbao: Mensajero.

Bisquerra, R. (2000). Educación emocionaly bienestar. Barcelona: CISSPRAXIS.

Cárdenas, C. S. (2008). Identificación de tipologías de actitud hacia las matemática en estudiantes de séptimo y octavo grados de educación primaria. Perfiles Educativos, $X X X(122)$, 94-108. Extraído el 25 Abril, 2010 de http://redalyc.uaemex.mx/redalyc/ pdf/132/13211181005.pdf

Cascante, A. y Salazar, C. (1993). Estrés, apoyo social y desempeño académico en estudiantes de 8vo, 9no y 10mo nivel de un colegio de San José. Tesis de Licenciatura para la obtención del título de Licenciada en Psicología. Universidad de Costa Rica. San José, Costa Rica. 
Cerezo, M. T., y Casanova, P. F. (2007). Diferencias de género en la motivación académicadelosalumnosdeeducación secundaria obligatoria. Recuperado el 24 de enero de 2008 de http:// www.investigacionpsicopedagogica. org/revista/articulos/3/espannol/ Art_3_31.pdf

Definición.de. (2008). Definición de actitud. Extraído el 25 Abril, 2010 de http:// definicion.de/actitud/

de Mézerville, G. (2004). Ejes de salud mental. Los procesos de autoestima, dar y recibir afecto y adaptación al estrés. México: Trillas.

Dumont, M., LeClerc, D., y Deslandes, R. (2003). Ressources personnelles et détresse psychologique en lien avec le rendement scolaire et le stress chez des élèves de quatrième secondaire. [Recursos personales y angustia psicológica en relación con el rendimiento escolar y el estrés en los alumnos de cuarto de secundaria]. Canadian Journal of Behavioral Science, 35(4), 254-267.

Ellis, A. (2000). Vivir en una sociedad irracional. Una guía para el bienestar mediante la terapia racional emotivoconductual. Barcelona: Paidós.

García, A. E. (2008). Motivación individual. Extraído el 7 Junio, 2008 de http://www.universidadabierta. edu.mx/Biblio/G/Garcia\% 20 AlfredoMotivacion\%20individual. htm

Gómez, M. (2005). Elementos de estadística descriptiva. San José: EUNED.

Güell, M., y Muñoz, J. (2000). Desconócete a ti mismo. Programa de alfabetización emocional. Barcelona: Paidós.
Hampton, D., Summer, Ch., y Webber, R. (1989). Manual de desarrollo de recursos humanos. México: Trillas.

Harter, S. (1999). The construction of the self. New York: Guilford.

Hernández, R. (1998). Metodología de la investigación. México: Mac Graw Hill.

Jackson, Y., y Frick, P. (1998). Negative life events and the adjustment of school age children: Testing protective models [Eventos de vida negativos y el ajuste de los niños en edad escolar: probando modelos protectores]. Journal of Clinical Child Psychology, 27(4), 370-380.

Jadue, G. (2001). Algunos efectos de la ansiedad en el rendimiento escolar. Estudios pedagógicos 27, 111-118. Extraído el 14 Abril, 2007 de http:// www.scielo.cl/scielo.php?pid=S07180 7052001000100008\&script=sci_arttext

Krauskopf, D. (1989). Adolescencia y educación en Costa Rica. San José: EUNED.

Martínez, E. S., y Díaz, D. A. (2007). Una aproximación psicosocial al estrés escolar. Educación y Educadores 10(2), 11-22. Extraído el 20 Agosto, 2008 de redalyc.uaemex.mx/redalyc/ pdf/834/83410203.pdf

Melgosa, J. (1995). Nuevo estilo de vida. iSin estrés! Madrid: Safeliz.

Molina, M., Baldares, T., y Maya, A. (1996). Fortalezcamos la autoestima y las relaciones interpersonales. San José: Impresos Sibaja.

Naranjo,M.L.(2004).Enfoquesconductistas, cognitivos y racional-emotivos. San 
José: Editorial Universidad de Costa Rica.

Neidhardt, J., Weinstein, M., y Conry, R. (1989). Seis programas para prevenir y controlar el estrés. Madrid: Deusto.

Newcomer, P. (1993). Cómo enseñar a los niños perturbados. México D. F.: FEC.

Oblitas, L. (2004). Psicología de la salud y calidad de vida. México:International Thompson Editores.

Océano. (1998). Enciclopedia de la Psicología (Vol. 4). España: Editorial Océano.

Rice, P. (2000). Adolescencia. Desarrollo, relaciones y cultura. Madrid: Prentice Hall.

Riso, W. (2002). Cuestión de dignidad. Aprenda a decir no y gane autoestima siendo asertivo. Bogotá: Norma.

Rogers, C. (1998). Terapia, personalidad y relaciones interpersonales. Buenos Aires: Nueva Visión.
Santrock, J. (2002). Psicología de la educación. México: Mc Graw Hill.

Trechera, J. L. (2005). Saber motivar: ¿el palo o la zanahoria? Extraído el 15 Febrero, 2008 de http://www. psicologia-online.com/articulos/2005/ motivacion.shtml

Universidad de Sevilla. (2005). Programa Golden 5: Una Intervención psicoeducativa. Extraído el 14 Abril, 2007 de http://www.golden5.org/golden5

Valdés, C. (2005, Agosto). Motivación. GestioPolis com. Extraído el 12 Noviembre, 2008 de http://www. gestiopolis.com/canales $5 / \mathrm{rrhh} /$ lamotici.htm

Valera, S. (2001). Curso de psicología ambiental. Universidad de Barcelona, España. Recuperado el 16 de mayo de 2007 de www.ub.es/ dppsa/psicamb/3460d.htm

Zeledón, M. y Chavarría, E. (2001). Educación infantil en valores desde la ética de la alteridad. San José: EUNED. 
